\title{
Acute Mastoiditis Following Leech Therapy in the Treatment of Acute Otitis Media
}

\author{
Coban $\mathrm{K}^{1^{*}}$, Bas $\mathrm{C}^{1}$ and Aydin $\mathrm{E}^{2}$ \\ ${ }^{1}$ Department of Otorhinolaryngology, Başkent University Alanya Research and Application Center, Antalya, Turkey \\ ${ }^{2}$ Department of Otorhinolaryngology, Başkent University Ankara Hospital, Ankara, Turkey
}

${ }^{*}$ Corresponding author: Coban K, Department of Otorhinolaryngology, Başkent University Alanya Research and Application Center, Antalya, Turkey, Tel: +90 53272541 96, E-mail: kubracob81@gmail.com Citation: Coban K, Bas C, Aydin E (2018) Acute Mastoiditis Following Leech Therapy in the Treatment of Acute Otitis Media. J Otolaryngol Res 1: 104

Article history: Received: 16 May 2018, Accepted: 18 June 2018, Published: 20 June 2018

\begin{abstract}
Otitis media is defined as an infection of the middle ear fluid and is the second most common pediatric diagnosis in the emergency department following upper respiratory infections. Acute mastoiditis in children develops when acute otitis media (AOM) spreads into the mastoid air cells inside the temporal bone. We present a 7 years old female child who attended to our clinic with severe symptoms of acute otitis media complicated with acute mastoiditis, to whom leech therapy was performed as the initial treatment, elsewhere. Due to the delay of the medical therapy, the disease deteriorated. Medicinal leech therapy (MLT) or hirudotherapy, an old technique, is believed to have possible positive effects on various conditions such as inflammatory and infectious diseases. They are used for their analgesic, anti-inflammatory, platelet inhibitory, anticoagulant, and thrombin regulatory functions. MLT is not an alternative, but may be a complementary and/or integrative choice. Deprivation or deferment of the exact medical treatment may lead to many undesirable complications.
\end{abstract}

Keywords: Acute Otitis Media; Acute Mastoiditis; Leech Therapy; Hirudotherapy

\section{Introduction}

Hırudotherapy (Leech therapy) was first used by Avicenna in 1020 [1]. It was believed that Hirudo medicinalis balanced the 'biological humours' by removing blood from patients. In $12^{\text {th }}$ century, Abd-el-latifal-Baghdadi claimed that leeches could help to clean tissues post operatively [1].

Leech therapy is used to reduce blood coagulation, relieving venous pressure, and stimulate blood circulation [1,2]. Moreover, leech saliva contains analgesic and anti-inflammatory substances, as well as having antimicrobial properties against E.coli, P.aeruginosa, B.cereus, S. Typhy and S.Aureus, C.albicans and C. neoformans $[1,3]$.

However in blood coagulation disorders, severe anemia, allergy to leech saliva, pregnancy and frailty, Hirudotherapy is contrindicated [3].

Leech therapy may have beneficial therapeutic actions, but it also has complications. The major complication of leech therapy is infection. The commonly encountered bacteria are Aeromonas spp., Pseudomonas spp., and Vibrio spp. Gunawan et. al. recommend prophylaxis [3].

\section{Case Report}

A 7 years of female child attended to our clinic with a complaint of left ear pain. In patient history, leech therapy was initially performed in another city. They referred to our clinic with persistent severe ear pain for nearly three weeks. The physical examination showed the three loci of entrance of leeches to the left ear (Figure 1). In her otoscopic examination, the left tympanic membrane was hyperemic and thickened. The post-auricular region was tender with palpation. Tympanometry showed a 'type B graphic with increased negative pressure, decreased compliance. Computerized tomography of the temporal bone (CT) reported dense fluid accumulation in the middle ear and mastoid air cells, referring to acute otitis media with acute mastoiditis (Figure 2 and 3 ). The 
patient was hospitalized, inmediate intravenous (IV) antibiotics (Ceftriaxone $2 x 750 \mathrm{mg}$ ) and anti-inflammatory-analgesic agents were administered for 10 days. Following the IV therapy, the patients' symptoms releived, she was discharged and oral Cefuroxime axetil $2 \times 250 \mathrm{mg}$ for 10 days was prescribed.

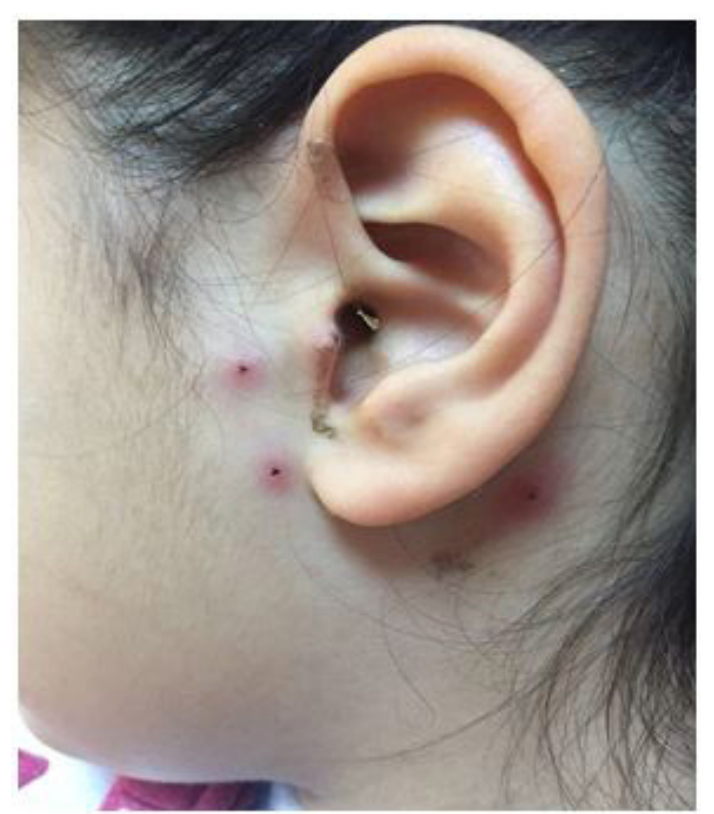

Figure 1: The appereance of the left ear. The hyperemic loci of the entrance of leeches are visible

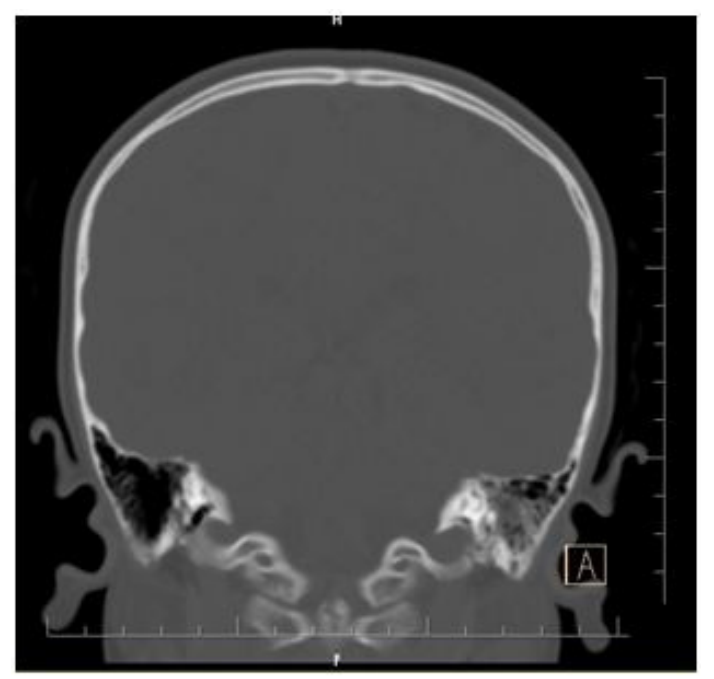

Figure 2: The mastoid cavity with fluid accumulation is demonstrated in CT coronal image

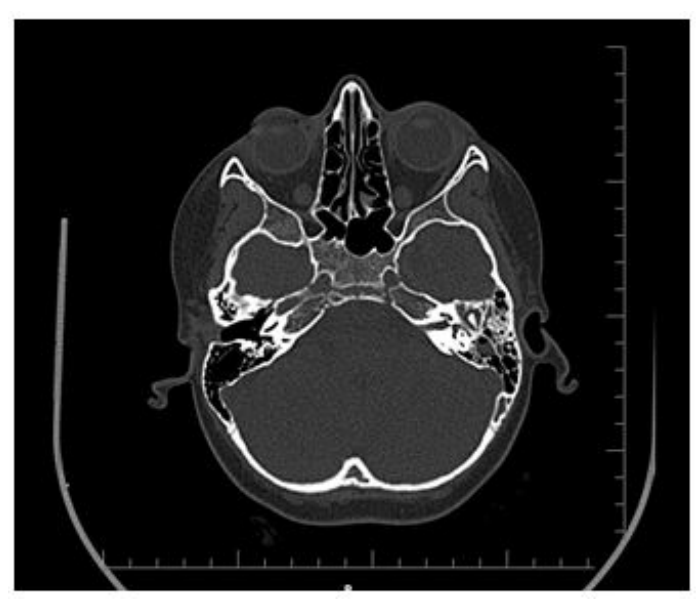

Figure 3: The axial CT image of the patient 


\section{Discussion}

AOM is a common disorder of early childhood which is also one of the most common reasons for referral of a child to an otolaryngology clinic. It is usually caused by the upper respiratory tract infections in which, the Eustachian tube is obstructed. Negative pressure occurs in the middle ear and via this pressure disequilibirium, the microbe-containing secretions from the upper airway mucousa, moves easiliy into the middle ear. The pathogens start to replicate in the middle ear and infection occurs [4-6]. A study of middle ear fluid in 79 children showed that $66 \%$ had bacteria and viruses, $27 \%$ had only bacteria, and $4 \%$ had only viruses [7]. The most common bacteria that cause AOM are, Streptococcus pneumoniae, Haemophilus influenza, and Moraxella catarrhalis $[8]$.

AOM can proceed to severe complications. Acute mastoiditis is one of them. In severe cases especially when antrum is blocked with musousal edema or granulation tissue, the drainage of the purulent secretions from the mastoid cells is interrupted. Thus the bacterial proliferation continues and with the penetration of mucousa, acute mastoiditis occurs. If the infectious process spreads to mastoid emissary veins and into the periostium, the first stage of acute mastoiditis is observed. If infiltration and demineralization of bone and degradation of its organic components occurs, the second stage (acute coalescent mastoiditis) is encountered [9,10].

Treatment strategies may vary among medical disciplines. A study showed that there was a decrease in use of antibiotics by otolaryngologists and pediatricians in between 2002 and 2009, while there was an increase in use by familiy practitioners in the same period [11].

The decision of whether or not to treat with prompt antibiotics is commonly based on age, severity of symptoms, the presence of otorrhea and laterality [4]. American Academy of Pediatrics (AAP) practice guideline recommends the use of antibiotics in patients with AOM, younger than 6 months, and for all children 6 months to two years of age with otorrhea and AOM; unilateral or bilateral AOM with severe symptoms, and bilateral AOM without otorrhea [9]. The decision to observe the patient without initial antibiotherapy should be made in accordance with caregivers and a close follow-up in every 48-72 hours is needed, so that antibiotics can be started in persistent or worsening conditions [12].

In this case, the patient was not followed-up closely and the symptoms and signs aggravated leading to acute mastoiditis. For this case leech therapy seems to delay the efficient medical therapies and with the lack of a close follow-up, the worsening of the condition was overlooked. However Hirudotherapy may also cause infections thus when performed, the patient should also be on probation of an experienced medical staff.

CT is the best radiologic option to evaluate the mastoid cavity and search for mastoid coalescence, abscess, mastoid cortex dehiscence [9]. If there is soft tissue edema or erythema in the absence of such radiological findings, hospitalization and admimistration of broad-spectrum antibiotics are suggested [9]. If there is no improvement of signs and symptoms in the following 24 hours, myringotomy and tube placement may be considered [9].

In our case the patient had otalgia, and tenderness in the mastoid region. The CT showed intense fluid in the mastoid cavity. As with medical therapy the symptoms and signs improved significantly, additional surgical management was not required.

\section{Conclusion}

Hirudotherapy may have potential anti-microbial, analgesic anti-inflammatory effects, however it may also cause secondary infections. In addition, this therapy is not routinely used in hospitals, and to our knowledge performed by alternative medicine therapists. Thus, applying leech to the infected area, may be time consuming and avoid a close follow-up by a clinician which may lead to a delay of the implementation of required medical and surgical therapies and cause serious complications.

\section{References}

1. Singh AP (2010) Medicinal leech therapy (hirudotherapy): a brief overview. Complement Ther Clin Pract 16: 213-5.

2. Godfrey K (1997) Uses of leeches and leech saliva in clinical practice. Nurs Times 93: 62-3.

3. Gunawan F, Wibowo YR, Bunawan NC, Turner JH (2015) Controversy: hirudotherapy (leech therapy) as an alternative treatment for osteoarthritis. Acta Med Indones 47: 176-80.

4. Rettig E, Tunkel DE (2014) Contemporary concepts in management of acute otitis media in children. Otolaryngol Clin North Am 47: 651-72.

5. Rovers MM, Schilder AG, Zielhuis GA, Rosenfeld RM (2004) Otitis media. Lancet 363: 465-73.

6. Chonmaitree T, Heikkinen T (1997) Role of viruses in middle-ear disease. Ann N Y Acad Sci 830: 143-57.

7. Ruohola A, Meurman O, Nikkari S, Skottman T, Salmi A, et al. (2006) Microbiology of acute otitis media in children with tympanostomy tubes: prevalences of bacteria and viruses. Clin Infect Dis 43: 1417-22.

8. Casey JR, Adlowitz DG, Pichichero ME (2010) New patterns in the otopathogens causing acute otitis media six to eight years after introduction of pneumococcal conjugate vaccine. Pediatr Infect Dis J 29: 304-9.

9. Lin HW, Shargorodsky J, Gopen Q (2010) Clinical strategies for the management of acute mastoiditis in the pediatric population. Clin Pediatr (Phila) 49: 110-5. 10. Rosen A, Ophir D, Marshak G (1986) Acute mastoiditis: a review of 69 cases. Ann Otol Rhinol Laryngol 95: 222-4.

11. Grossman Z, Silverman BG, Miron D (2013) Physician specialty is associated with adherence to treatment guidelines for acute otitis media in children. Acta Paediatr 102: 29-33.

12. Lieberthal AS, Carroll AE, Chonmaitree T, Ganiats TG, Hoberman A et al. (2013) The diagnosis and management of acute otitis media. Pediatrics. 131: 964-99. 